\title{
Alloyed-core colloidal quantum dot DFB laser with encapsulated gain region
}

\author{
L. McLellan, B. Guilhabert, N. Laurand and M. D. Dawson \\ Institute of Photonics, University of Strathclyde, Glasgow G4 0NW, UK \\ Email: luke.mclellan@strath.ac.uk
}

\begin{abstract}
A CdS $\mathrm{Se}_{1-\mathrm{x}} / \mathrm{ZnS}$ colloidal quantum dot distributed-feedback laser operating in the nanosecondregime with a threshold below $3 \mathrm{~kW} / \mathrm{cm}^{2}$ is reported. The laser emits vertically up to $40 \mathrm{~nJ}$ at $600 \mathrm{~nm}$ with an efficiency of $1.2 \%$.

Introduction: Colloidal quantum dots (CQDs) are inorganic semiconductor nanocrystals that represent an attractive material for visible-wavelength laser technology. CQDs combine the quantum confinement effect desirable for wavelength versatility and efficient laser operation with solution-processing capabilities that enable great flexibility for device fabrication. A wide range of optically pumped CQD lasers has thus been demonstrated over recent years, including in mechanically-flexible formats [1-6]. While earlier CQD laser demonstrations made use of ultra-fast optical excitation (fs to few ps), the focus is now on closing the gap to real-world applications with compact systems [5]. This means improving the laser performance and establishing the technology for longer pulse operation, from the ns [4-6] to the continuous-wave regime, so that stable diode-pumping operation becomes possible. In this work, we utilise yellow-orange alloyed core/shell $\mathrm{CdS}_{\mathrm{x}} \mathrm{Se}_{1-\mathrm{x}} / \mathrm{ZnS} \mathrm{CQDs}$ as the gain region of a bilayer verticallyemitting distributed-feedback (DFB) laser. Under 5ns-long pulse excitation at $355 \mathrm{~nm}$, this laser emits up to $40 \mathrm{~nJ}$ at $600 \mathrm{~nm}$, limited by the pump energy. Crucially, the threshold is below $3 \mathrm{~kW} / \mathrm{cm}^{2}$, to our knowledge the lowest threshold for a CQD laser operating in such temporal regime.
\end{abstract}

Device fabrication and experimental setup: The design of the DFB laser is shown in the schematic of Fig. 1. It consists of a 300nm CQD film on top of a surface-patterned glass substrate overcoated with a $180 \mathrm{~nm}$ PVA layer. The pattern is a rectangular grating with a $380 \mathrm{~nm}$ periodicity. The PVA acts as an encapsulant that symmetrises the refractive index profile of the overall laser structure. This design increases the laser mode overlap with the CQD region and therefore increases the modal gain. Alloyedcore/shell $\mathrm{CdS}_{\mathrm{x}} \mathrm{Se}_{1-\mathrm{x}} / \mathrm{ZnS}$ CQDs (Trilite ${ }^{\mathrm{TM}}$ from Cytodiagnostics) with an intrinsic photoluminescence (PL) centred at $575 \mathrm{~nm}$ were used. Auger recombination is known to be a limiting factor for laser performance in spherical CQDs with abrupt core/shell interfaces and the detrimental effect is exacerbated in CQDs of small size [7]. Alloying of the core enables the use of CQDs with relatively large size (here typically $6 \mathrm{~nm}$ ) whatever the emission wavelength $(450 \mathrm{~nm}$ to $665 \mathrm{~nm})$ while alleviating the issue of the large and abrupt core/shell energy interface. The CQDs were initially in toluene at a concentration of $1 \mathrm{mg} \cdot \mathrm{mL}^{-1}$. The toluene was evaporated under vacuum before the CQDs in powder form were re-diluted in a mix of 1.6:1 PMMA in chloroform. This solution was then spun cast onto the glass substrate. After deposition of the PVA the sample was annealed at $30^{\circ} \mathrm{C}$ for 120 hours. The amplified spontaneous emission (ASE) characteristics of the gain structure were measured independently of the laser cavity by depositing the same CQD and PVA layers onto a smooth glass substrate (CQD/PVA sample). Another sample on smooth glass but without the PVA layer was also characterized (CQD sample) to verify the effect of the latter. Samples were optically pumped with an Nd:YAG laser, emitting at $355 \mathrm{~nm}$ with $5 \mathrm{~ns}$ pulses and a repetition rate of $10 \mathrm{~Hz}$. The ASE samples were excited with a $1.5 \mathrm{~mm} \times 4.4 \mathrm{~mm}$ stripe and the edge PL was collected for analysis. The CQD sample's edge PL was also measured under CWexcitation from a $10 \mathrm{~mW}, 371 \mathrm{~nm}$ laser diode with a $6.1 \mathrm{~mm} \times 1.7 \mathrm{~mm}$ stripe. The DFB laser was excited with a $0.74 \mathrm{~mm} \times 4.4 \mathrm{~mm}$ pump stripe and the vertical laser emission was collected for analysis. 
Results and discussion: Fig. 1 (a) is an image of the CQD laser above threshold. Figure 2a shows the normalized edge emission of the ASE samples (CQD and CQD/PVA) pumped at a level above the onset of stimulated emission. The edge emission spectrum of the CQD/PVA sample under low intensity CWexcitation is also represented to act as the edge PL reference below the ASE threshold. Compared to the edge PL at $583 \mathrm{~nm}$, the ASE peak for the CQD sample is red-shifted by $6 \mathrm{~nm}$, which is attributed to the bi-excitonic binding energy (type I CQDs). We note that this binding energy is lower than for typical redemitting CdSe/CdS CQDs [5]. The ASE peak for the CQD/PVA sample is further red-shifted by $9 \mathrm{~nm}$. This further shift is caused by the higher modal confinement in the CQD region, exacerbating the effect of re-absorption of this quasi-three level laser material. The higher confinement though leads to a higher modal gain and the ASE threshold is lower for the CQD/PVA sample $\left(382 \mu \mathrm{J} / \mathrm{cm}^{2}\right.$ vs $815 \mu \mathrm{J} / \mathrm{cm}^{2}$, data not shown). Fig. 2b summarizes the performance of the equivalent CQD/PVA DFB laser. The laser emits principally in the $\mathrm{TE}_{0}$ mode at $600 \mathrm{~nm}$ but the $\mathrm{TM}_{0}$ mode can be seen at $597 \mathrm{~nm}$ at high pump level. The $\mathrm{TE}_{0}$ total linewidth is $0.45 \mathrm{~nm}$ due to the presence of lateral modes. The threshold is only $\sim 13.5 \mu \mathrm{J} / \mathrm{cm}^{2}$ $\left(<3 \mathrm{~kW} / \mathrm{cm}^{2}\right)$, on a par with some organic lasers operated in the same temporal regime. The laser energy for a pump at $3.7 \mathrm{~mJ}$ is $40 \mathrm{n} \mathrm{J}$ corresponding to an efficiency of $1.2 \%$.

Conclusion: We have reported a CQD DFB laser with what is to our knowledge an ultra-low threshold for the ns temporal regime. This performance is attributed to the nature of the alloyed core CQDs and to the bilayer design of the laser cavity.

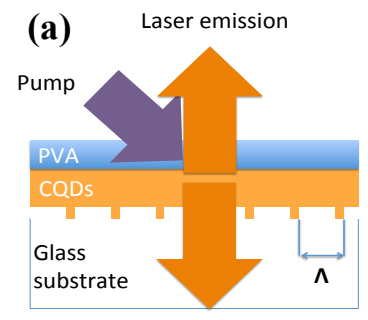

(b)
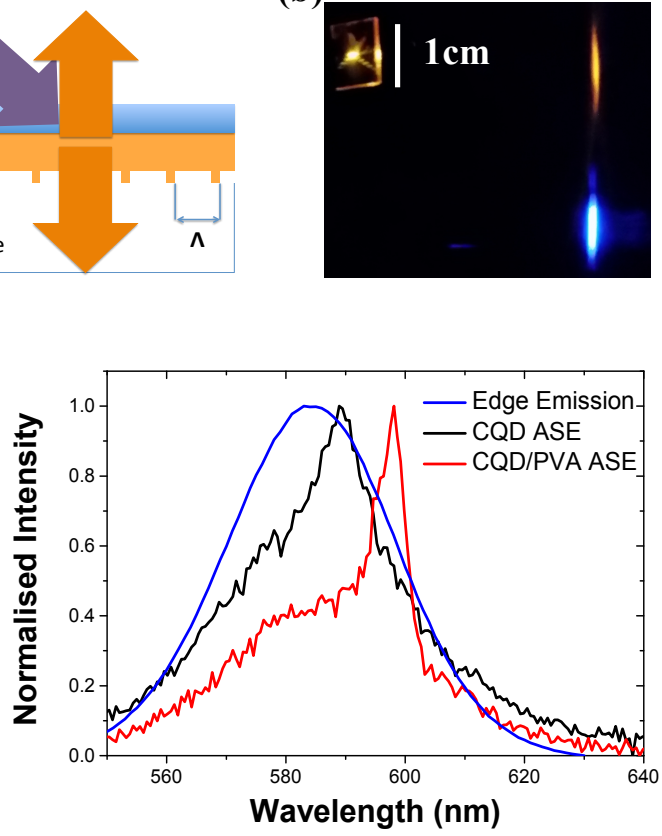

(a)$$
\text { (a) }
$$

Figure 1: (a) Schematic of the laser structure with grating substrate, a $\Lambda=380 \mathrm{~nm}$ CQD layer and a $180 \mathrm{~nm}$ PVA layer. The laser is pumped at an angle and the laser emits vertically. (b) Image of the CQD laser with its vertical emission (orange, top) and the diffracted pump beam (violet, bottom).

Figure 2: (a) Normalised spectra of the edge emission of alloyed - core/shell CQDs in a film compared ASE shift with and without encapsulation (b) Transfer function of PVA/CQD/DFB laser with optical pump source resulting in a threshold fluence $\sim 13.5 \mu \mathrm{J} / \mathrm{cm}^{2}$ and (inset) multimode laser spectrum with overall linewidth of $0.45 \mathrm{~nm}$.

[1] A. V. Malko et al., Appl. Phys. Lett. 81, 1303 (2002).

[2] S. Hoogland et al., Opt. Express 14, 3273 (2006).

[3] V. M. Menon et al., Opt. Express 16, 19535(2008).

[4] C. Dang et al., Nature Nanotechnology 7, 335 (2012).

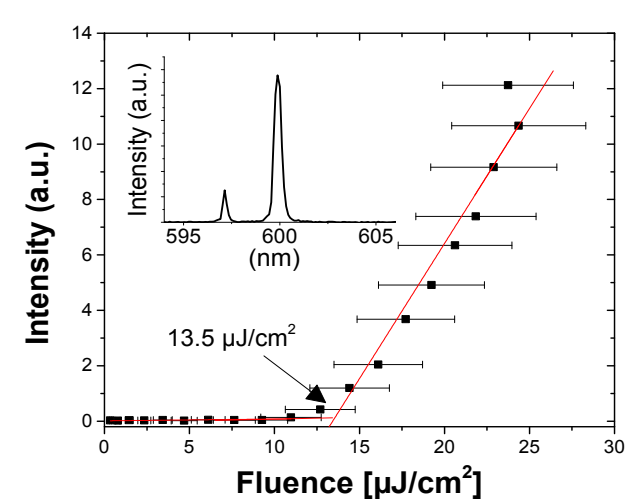

(b)
[5] B. Guilhabert et al., Opt. Express 22, 7308 (2014).

[6] C. Foucher et al., Appl. Phys. Lett. 104 (14), 141108 (2014).

[7] G. E. Cragg et al., Nano Lett. 10, 313 (2010). 